\title{
Improving Neuromuscular Monitoring and Reducing Residual Neuromuscular Blockade With E-Learning: Protocol for the Multicenter Interrupted Time Series INVERT Study
}

Jakob Louis Demant Thomsen ${ }^{1}$, MD; Ole Mathiesen², MD, PhD, Clinical Research Associate Professor; Daniel Hägi-Pedersen ${ }^{3}, \mathrm{MD}, \mathrm{PhD}$; Lene Theil Skovgaard ${ }^{4}$, Cand.stat., Associate Professor; Doris Østergaard ${ }^{5}, \mathrm{MD}, \mathrm{DMSc}$, Professor; Jens Engbaek ${ }^{1}$, DMSc, MD; Mona Ring Gätke ${ }^{1}, \mathrm{MD}, \mathrm{PhD}$

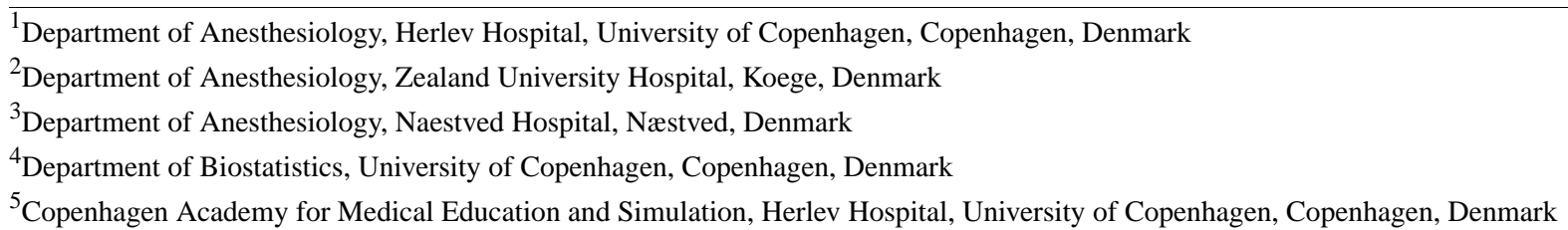

\section{Corresponding Author:}

Jakob Louis Demant Thomsen, MD

Department of Anesthesiology

Herlev Hospital

University of Copenhagen

Herlev Ringvej 75

Copenhagen, 2730

Denmark

Phone: 4525118646

Fax: 4544534806

Email: jakob.louis.thomsen@ regionh.dk

\section{Abstract}

Background: Muscle relaxants facilitate endotracheal intubation under general anesthesia and improve surgical conditions. Residual neuromuscular blockade occurs when the patient is still partially paralyzed when awakened after surgery. The condition is associated with subjective discomfort and an increased risk of respiratory complications. Use of an objective neuromuscular monitoring device may prevent residual block. Despite this, many anesthetists refrain from using the device. Efforts to increase the use of objective monitoring are time consuming and require the presence of expert personnel. A neuromuscular monitoring e-learning module might support consistent use of neuromuscular monitoring devices.

Objective: The aim of the study is to assess the effect of a neuromuscular monitoring e-learning module on anesthesia staff's use of objective neuromuscular monitoring and the incidence of residual neuromuscular blockade in surgical patients at 6 Danish teaching hospitals.

Methods: In this interrupted time series study, we are collecting data repeatedly, in consecutive 3-week periods, before and after the intervention, and we will analyze the effect using segmented regression analysis. Anesthesia departments in the Zealand Region of Denmark are included, and data from all patients receiving a muscle relaxant are collected from the anesthesia information management system MetaVision. We will assess the effect of the module on all levels of potential effect: staff's knowledge and skills, patient care practice, and patient outcomes. The primary outcome is use of neuromuscular monitoring in patients according to the type of muscle relaxant received. Secondary outcomes include last recorded train-of-four value, administration of reversal agents, and time to discharge from the postanesthesia care unit as well as a multiple-choice test to assess knowledge. The e-learning module was developed based on a needs assessment process, including focus group interviews, surveys, and expert opinions.

Results: The e-learning module was implemented in 6 anesthesia departments on 21 November 2016. Currently, we are collecting postintervention data. The final dataset will include data from more than 10,000 anesthesia procedures. We expect to publish the results in late 2017 or early 2018.

Conclusions: With a dataset consisting of thousands of general anesthesia procedures, the INVERT study will assess whether an e-learning module can increase anesthetists' use of neuromuscular monitoring. 
Trial Registration: Clinicaltrials.gov NCT02925143; https://clinicaltrials.gov/ct2/show/NCT02925143 (Archived by WebCite ${ }^{\circledR}$ at http://www.webcitation.org/6s50iTV2x)

(JMIR Res Protoc 2017;6(10):e192) doi: 10.2196/resprot.7527

\section{KEYWORDS}

e-learning; neuromuscular monitoring; objective neuromuscular monitoring; quantitative neuromuscular monitoring; residual neuromuscular blockade; protocol

\section{Introduction}

Muscle relaxants facilitate endotracheal intubation under general anesthesia and improve surgical conditions. Postoperative residual neuromuscular blockade, or residual blockade for short, occurs if the effects of the relaxant have not subsided or have not been sufficiently reversed before the patient is awakened [1]. Volunteer studies have shown partial paralysis to be associated with general muscle fatigue, double vision, and speech difficulty [2,3]. In clinical studies, residual blockade increased the risk of respiratory complications such as airway obstruction, hypoxemia, and tracheal reintubation as well as the risk of a prolonged length of stay in the postanesthesia care unit [4-7].

The depth of the neuromuscular blockade can be monitored intraoperatively with a neuromuscular monitoring device that measures the muscle response to peripheral nerve stimulation. Typically, the response of the adductor pollicis muscle, adducting the thumb, is measured using an acceleromyographic neuromuscular monitor following stimulation of the ulnar nerve at the level of the wrist [8]. In clinical studies, this type of objective neuromuscular monitoring reduced the incidence of residual blockade and symptoms of muscle weakness [9-11]. Hence, for more than a decade, experts in the field have recommended routine neuromuscular monitoring when administering muscle relaxants [12-15]. Nevertheless, surveys reveal that many anesthetists do not routinely apply neuromuscular monitoring, even if the equipment is available [16-18]. In particular, clinicians often refrain from applying neuromuscular monitoring when administering only the short-acting muscle relaxant succinylcholine because the paralytic effect subsides within 10 minutes in most, but not all, patients. However, it was recently confirmed that even patients receiving only succinylcholine are at risk of experiencing paralysis when awakened from anesthesia, especially if not monitored with a nerve stimulator [19,20].

Repeated local educational efforts in anesthesia departments may increase the use of neuromuscular monitoring and reduce the incidence of residual blockade [21,22]. As these efforts are time consuming and require the presence of expert personnel, it is relevant to consider if an e-learning module may provide an alternative method to increase the use of neuromuscular monitoring. E-learning can achieve results similar to traditional instructional methods, but few studies have assessed the impact of e-learning on patient outcomes, possibly because of the large sample size required [23].
The aim of the INVERT study is to assess the impact of an e-learning module on neuromuscular monitoring on the application frequency of objective neuromuscular monitoring and the incidence of residual neuromuscular blockade in patients monitored with a nerve stimulator.

We hypothesize that the e-learning module will increase the use of objective neuromuscular monitoring significantly in both patients receiving succinylcholine and patients receiving a nondepolarizing muscle relaxant.

\section{Methods}

\section{Design}

The study is designed to assess the effect of an e-learning module on all three of the proposed levels of medical education translational research: knowledge and skills, patient care practice, and patient outcomes [24]. A "dilution" of the effect should be expected in the process of changing clinicians' knowledge, in their behavior as well as actual patient outcomes [23]. Therefore, data from a large number of anesthesia procedures are likely required to obtain adequate strength. To make this feasible, we have chosen to conduct the study at all major hospitals in the Zealand Region of Denmark, where data on use of neuromuscular monitoring are automatically recorded in the anesthesia information management system MetaVision (iMDsoft ${ }^{\circledR}$, Düsseldorf, Germany). Furthermore, we have chosen the interrupted time series design, in which data are collected repeatedly, at fixed intervals, before and after the intervention (Figure 1).

The effect of the intervention is analyzed statistically using segmented regression analysis, testing for changes in both the level and the trend of the outcome. The design is proposed to be the strongest quasi-experimental approach for assessing the longitudinal effect of interventions and also allows description of the timing of the effect of the intervention [25]. This design is well suited for our setting, where the number of participating departments is limited to the 6 departments of anesthesia at the teaching hospitals in the Zealand Region of Denmark, making experimental designs such as cluster randomized trials unfeasible [26]. Baseline data are being obtained from the descriptive study "Use of neuromuscular blocking agents and neuromuscular monitoring in 7 Danish teaching hospitals-a cross-sectional study" (NCT02914119), in which data are collected from the same hospitals in the year leading up to the intervention. The e-learning module was implemented over a period of 2 weeks, and we area again collecting data from all departments at fixed 3-week intervals. 
Figure 1. Example of data collection for an interrupted time series study.

\begin{tabular}{|l|l|l|l|l|l|l|l|}
\hline Department 1 & & & & & & & \\
\hline Department 2 & & & & & & & \\
\hline Department 3 & & & & & & & \\
\hline Department 4 & & & & & & & \\
\hline Department 5 & & & & & & & \\
\hline Department 6 & & & & & & & \\
\hline
\end{tabular}

Baseline data collection periods ( 3 weeks each)

Implementation of intervention (2 weeks)

$\square$ Postintervention data collection periods ( 3 weeks each)

\section{Eligibility}

\section{Departments}

We are including the 6 anesthesia departments at the teaching hospitals in the Zealand Region of Denmark following agreement of the head of each department to participate.

\section{Anesthesia Personnel}

We are including the following anesthesia personnel: nurse anesthetists in training, certified registered nurse anesthetists, first- though fourth-year residents in anesthesiology, and certified anesthesiologists. We are excluding personnel without clinical functions, such as administrative personnel.

\section{Patients}

We are including and collecting data from all patients undergoing general anesthesia with neuromuscular blockade in each data collection period. Patients undergoing general anesthesia on more than one occasion will be included in the analyses as one case for every general anesthetic received.

\section{Intervention: E-Learning Module}

The investigator group has developed the e-learning module in collaboration with the Regional Unit for Development and
Evaluation of Learning Technologies in the Capital Region of Denmark. Learning objectives for the module are based on a needs assessment, including focus group interviews with nurse anesthetists and anesthesiologists from 5 different Danish hospitals and clinical observations of neuromuscular monitoring use in practice (NCT02239965) as well as a previous Danish survey [18] and expert opinions. The main topics in the course are as follows: (1) background and clinical consequences of residual blockade, (2) neuromuscular monitoring and stimulation patterns, (3) practical tips on monitoring and troubleshooting equipment malfunction, and (4) reversal of neuromuscular blockade. The course participants are of different educational backgrounds and have anesthesia experience ranging from short periods to decades of employment. We have aimed to target this diversity in participant background by making the e-learning module adaptable to learners' needs, specifically by making part of the material optional (Figure 2) [27]. Furthermore, clickable animations are included to stimulate interaction and increase learning [28], while examples of monitor output make the module relevant to participants' daily practice. The course duration is approximately 30 minutes.

Figure 2. Screen shot from e-learning module.

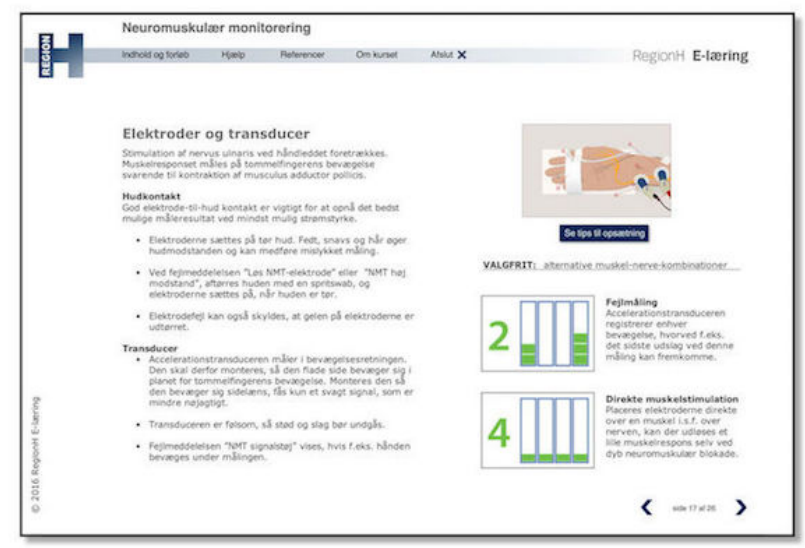

Interactive animations

Optional content accessed by

clicking on hyperlink

Examples of monitor output to

increase relevance to clinicians'

daily practice 


\section{Implementation of the Intervention}

We recruited a local investigator at each study site. This person is responsible for motivating the anesthesia personnel to complete the e-learning module and introduce all new employees to it. On the first day of implementation, the local investigators introduced the e-learning module. The primary investigator also visited the departments in the intervention period and introduced the study, but without revealing the primary outcome to the participants. The participating departments ensured that the personnel could complete the module during their normal work hours, such as by having colleagues cover for them while they complete the module. Course progress was logged for each anesthetist, and email reminders were sent to ensure the highest possible completion rate. The local investigator and the head of department were also informed about the completion rate throughout the implementation period. To increase awareness about the study and the e-learning module, the local investigators distributed candies and pens with the INVERT study logo at intervals. New employees joining the departments after the intervention period were also included in the study. The module is available for ongoing access, meaning that learners can still access the module, such as for use as a clinical troubleshooting tool. All anesthetic practices and medication use are at the discretion of the individual anesthetist according to local guidelines.

\section{Blinding}

Given the nature of the intervention, it was not possible to blind the anesthesia personnel. However, they are not informed of the specific outcomes, only that the study "aims to assess the effect of the e-learning module on use of neuromuscular monitoring". The investigators in charge of data analysis will not have access to the data until after the final data collection period.

\section{Outcomes}

We will assess the effect of the e-learning module on knowledge, behavior, and patient outcomes. A meta-analysis of studies on the effect of Web-based learning indicates that an e-learning module will have greatest effect on knowledge, a smaller effect on skills, and a yet smaller effect on behavior and patient-related outcomes [23]. We have chosen the primary outcome based on what we believe to be of highest clinical relevance, balanced with the feasibility of collecting data on this outcome from thousands of patients.

\section{Primary Outcome}

- Application of neuromuscular monitoring in cases involving succinylcholine only

- Application of neuromuscular monitoring in cases involving a nondepolarizing muscle relaxant (with or without succinylcholine)

The primary outcome is divided because the two types of relaxants are used in different clinical situations: succinylcholine for "rapid sequence induction and intubation" and nondepolarizing relaxants for nonemergent tracheal intubation.

\section{Secondary Outcomes}

- Last recorded train-of-four (TOF) ratio (a stimulation pattern commonly used in neuromuscular monitoring, where a ratio $<0.9$ is defined as residual blockade) before tracheal extubation in patients receiving a nondepolarizing relaxant

- Administration of the reversal agent sugammadex or neostigmine and repeated administration of the reversal agent

- Timing and dosage of reversal with sugammadex or neostigmine in relation to the neuromuscular monitoring values

- Time from administration of the reversal agent (sugammadex or neostigmine) to extubation

- Time from tracheal extubation or removal of the supraglottic airway device to discharge from the postanesthesia care unit in cases involving a nondepolarizing relaxant with or without neuromuscular monitoring

- Pre- and postcourse test scores as a measure of course participants' knowledge

Secondary analyses that may be performed if the data allow it include the incidence of respiratory complications, arterial oxygen desaturation, and the effect of the e-learning module at the individual level.

\section{Data Collection}

Data are recorded in the MetaVision anesthesia information management system either automatically or manually, by the anesthetist. TOF data are recorded automatically when the neuromuscular monitoring module is activated. The anesthesia information management system saves TOF data every minute, even if the neuromuscular monitoring module measures a TOF value every 12 seconds. To ensure that the reported TOF values at the time of tracheal extubation are not erroneously measured due to lack of fixation of the patient's hand, movements caused by the surgical personnel, or other factors, we will collect the last five TOF values before tracheal extubation and use the highest value, given that the values increase incrementally. We will develop an algorithm for choosing the last correctly measured TOF value and will perform manual validation on part of the dataset. Data manually entered into the anesthesia information management system include the type and dose of intravenous drugs and the time of tracheal extubation. The anesthetist enters these data by pressing a button in the software and can alter the time and the details, such as the dose of medicine administered, afterward if needed. Where possible, we will seek to validate these manually entered data, such as by comparing them to changes in ventilatory data from the anesthesia machine. In case of doubt about the correctness of data for a particular case, we will access the full dataset for that case in order to investigate further.

\section{Sample Size Calculation and Statistical Analysis}

A simulation-based sample size calculation revealed that the sample size necessary to detect an increase in neuromuscular monitoring from $40 \%-60 \%$ (with an $80 \%$ power) would require 3 consecutive data collection periods, each comprising approximately 100 patients per site, both before and after the implementation of the intervention. We expected to achieve 
this sample size by letting each period be 3 weeks long. The baseline value of $40 \%$ was based on a 1-month data sample from quality assurance at the participating hospitals, while the increase to $60 \%$ reflects the minimal effect size that we believe would make it worthwhile to disseminate the course to other departments. It is possible that the effect of the module could "wear off" after the 9 weeks of postintervention data collection if either a loss of knowledge occurs or the motivation to use neuromuscular monitoring decreases after the study period. To assess this, we extended the postintervention data collection period beyond what the sample size calculation indicated. This extension is ethically justifiable because prolonging the data collection does not affect patient treatment and because the data are already automatically collected in the anesthesia information management system.

When analyzing the data, we will use segmented regression analysis [25]. All results will be reported with $95 \%$ confidence intervals when relevant. We will test continuous variables for normality by visual inspection before summarization with the mean (standard deviation). Non-normally distributed continuous variables and ordinal variables will be described as the median (95th percentile range) and categorical variables as the number (percentage), if not otherwise specified. Analyses will be performed using SPSS version 22 (SPSS Inc., Chicago, IL, USA). The investigators JLT and LTS will conduct the analyses. We will consider a two-tailed $P$ value of less than 0.05 to be statistically significant.

\section{Missing Data}

In the analysis of the secondary outcomes, we will exclude cases with missing data on the outcome studied, that is, we will perform only per-protocol analyses. However, in reporting of the median TOF ratio at tracheal extubation, we will include both an analysis where a TOF count between 0 and 3 (and hence no TOF ratio reported) is included as a TOF ratio of 0 and a per-protocol analysis where only cases with a TOF ratio are included. Furthermore, we will report the proportion of patients transferred from the operating room while still tracheally intubated and, if possible, report the cause for this, such as severe residual neuromuscular blockade.

\section{Ethical Considerations and Study Registration}

The study solely uses data routinely registered in the anesthesia information management system. The Committees on Health Research Ethics for the Capital Region of Denmark have confirmed that the study does not need approval from the committee system and that there is no need for individual patient consent (protocol no. 16028220). The study is registered at clinicaltrials.gov (NCT02925143) and with The Danish Data Protection Agency (HGH-2015-063/04364).

\section{Results}

All 6 anesthesia departments agreed to participate in the study. The e-learning module was implemented simultaneously in all departments on 21 November 2016. Currently, postintervention data are being collected. We expect to publish the results in late 2017 or early 2018.

\section{Discussion}

We have designed a study that aims to assess the value of an e-learning module on neuromuscular monitoring at all levels of potential effect: knowledge and skills, patient care practice, and patient outcomes. We expect that the multicenter design and the large number of participants completing the e-learning module will increase the internal and external validity of results. The primary outcomes are automatically recorded in the anesthesia information management system, increasing the validity of the collected data.

While randomized controlled trials are the gold standard of design in clinical research, this design is sometimes unfeasible in medical education research. Participants, in our case anesthesia personnel, completing the e-learning module cannot be randomized individually to use or not use the knowledge that they gained from the module. Similarly, while it is practically possible to randomize patients at a particular hospital to receive treatment from either an anesthetist who had completed the module or one who had not, there would be a nesting effect that could affect the results, such as due to local traditions regarding neuromuscular monitoring. The effect of the intervention could also spill over from anesthetists in the intervention group to the control group, such as through teamwork or meetings. One way of overcoming this challenge would be to conduct a cluster randomized trial [26], but, as mentioned earlier, this was not possible because of the limited number of potential participating departments. It is important to note, however, that the INVERT study aims to answer the question "can use of neuromuscular monitoring be increased by implementing an e-learning module on the subject?", as opposed to assessing the effect of e-learning in general. Therefore, the interrupted time series design may very well be the optimum choice of design [25]. Furthermore, the baseline study describing use of neuromuscular monitoring in the participating departments in the year leading up to implementation of the e-learning module may, to some degree, account for not having a control group by describing an underlying trend in use of monitoring (ie, the departments function as their own historical controls).

There will be some limitations to the study. The anesthetist manually enters certain data in the anesthesia information management system, which should be taken into account when interpreting the results. Studies reporting on the incidence of residual neuromuscular blockade typically measure the TOF ratio upon arrival to the postanesthesia care unit. As this approach would require the presence of investigators in all departments at all times throughout the study period, it was not possible in our study. Instead, we chose to use the data that were readily available from the anesthesia information system, namely, the last recorded TOF ratio in the operating room. For this reason, we will not obtain data on this secondary outcome for patients without monitoring with a nerve stimulator. Finally, we did not design the study to assess the effect of the e-learning module at the individual participant level, but rather at the levels of each department, as a whole. However, if possible with the data obtained, the former will described in a secondary analysis. 


\section{Perspectives}

One of the advantages of Web-based learning, such as an e-learning module, is that participant numbers can be increased with only small expenses [29]. If the e-learning module proves effective in increasing the use of neuromuscular monitoring, it may be implemented in all Danish anesthesia departments or in the training of anesthesiologists and nurse anesthetists both nationally and internationally.

In conclusion, with a dataset consisting of thousands of general anesthesia procedures, the INVERT study will thoroughly assess whether an e-learning module can increase anesthetists' use of neuromuscular monitoring.

\section{Acknowledgments}

The authors wish to thank Peter Hultberg, Rikke Holm Møller, Søren Brink, and Yoon Falk Jensen from the Regional Unit for Development and Evaluation of Learning Technologies in the Capital Region of Denmark for their work producing the e-learning module. Thanks also to the anesthesia departments as well as the anesthesia personnel for their participation in the study.

The study is supported in part by a research grant from the Investigator-Initiated Studies Program of Merck Sharp \& Dohme Corp. Before deciding to support the study, Merck Sharp \& Dohme Corp. reviewed the protocol. The e-learning module for this study was produced independently from Merck Sharp \& Dohme Corp. The opinions expressed in this protocol paper and the resulting paper are those of the authors and do not necessarily represent those of Merck Sharp \& Dohme Corp. Merck Sharp \& Dohme Corp was allowed to review this paper before submission but had no decisional right.

\section{Conflicts of Interest}

JLT received two research grants from the Investigator-Initiated Studies Program of Merck Sharp \& Dohme Corp. There were no other potential conflicts of interest.

MRG received research grants from the Investigator-Initiated Studies Program of Merck Sharp \& Dohme Corp and received speaker's fees from Merck.

The other authors had no conflict of interest to decare.

\section{References}

1. Murphy GS, Brull SJ. Residual neuromuscular block: lessons unlearned. Part I: definitions, incidence, and adverse physiologic effects of residual neuromuscular block. Anesth Analg 2010 Jul;111(1):120-128. [doi: 10.1213/ANE.0b013e3181da832d] [Medline: 20442260]

2. Kopman AF, Yee PS, Neuman GG. Relationship of the train-of-four fade ratio to clinical signs and symptoms of residual paralysis in awake volunteers. Anesthesiology 1997 Apr;86(4):765-771. [Medline: 9105219]

3. Eriksson LI, Sundman E, Olsson R, Nilsson L, Witt H, Ekberg O, et al. Functional assessment of the pharynx at rest and during swallowing in partially paralyzed humans: simultaneous videomanometry and mechanomyography of awake human volunteers. Anesthesiology 1997 Nov;87(5):1035-1043. [Medline: 9366453]

4. Murphy GS, Szokol JW, Marymont JH, Greenberg SB, Avram MJ, Vender JS. Residual neuromuscular blockade and critical respiratory events in the postanesthesia care unit. Anesth Analg 2008 Jul;107(1):130-137. [doi: 10.1213/ane.0b013e31816d1268] [Medline: 18635478]

5. Butterly A, Bittner EA, George E, Sandberg WS, Eikermann M, Schmidt U. Postoperative residual curarization from intermediate-acting neuromuscular blocking agents delays recovery room discharge. Br J Anaesth 2010 Sep;105(3):304-309. [doi: 10.1093/bja/aeq157] [Medline: 20576632]

6. Murphy GS, Szokol JW, Avram MJ, Greenberg SB, Shear T, Vender JS, et al. Postoperative residual neuromuscular blockade is associated with impaired clinical recovery. Anesth Analg 2013 Jul;117(1):133-141. [doi: 10.1213/ANE.0b013e3182742e75] [Medline: 23337416]

7. Grosse-Sundrup M, Henneman JP, Sandberg WS, Bateman BT, Uribe JV, Nguyen NT, et al. Intermediate acting non-depolarizing neuromuscular blocking agents and risk of postoperative respiratory complications: prospective propensity score matched cohort study. BMJ 2012 Oct 15;345:e6329 [FREE Full text] [Medline: 23077290]

8. Brull SJ, Murphy GS. Residual neuromuscular block: lessons unlearned. Part II: methods to reduce the risk of residual weakness. Anesth Analg 2010 Jul;111(1):129-140. [doi: 10.1213/ANE.0b013e3181da8312] [Medline: 20442261]

9. Murphy GS, Szokol JW, Avram MJ, Greenberg SB, Marymont JH, Vender JS, et al. Intraoperative acceleromyography monitoring reduces symptoms of muscle weakness and improves quality of recovery in the early postoperative period. Anesthesiology 2011 Nov;115(5):946-954. [doi: 10.1097/ALN.0b013e3182342840] [Medline: 21946094]

10. Gätke MR, Viby-Mogensen J, Rosenstock C, Jensen FS, Skovgaard LT. Postoperative muscle paralysis after rocuronium: less residual block when acceleromyography is used. Acta Anaesthesiol Scand 2002 Feb;46(2):207-213. [Medline: 11942873]

11. Murphy GS, Szokol JW, Marymont JH, Greenberg SB, Avram MJ, Vender JS, et al. Intraoperative acceleromyographic monitoring reduces the risk of residual neuromuscular blockade and adverse respiratory events in the postanesthesia care unit. Anesthesiology 2008 Sep;109(3):389-398. [doi: 10.1097/ALN.0b013e318182af3b] [Medline: 18719436]

12. Eriksson LI. Evidence-based practice and neuromuscular monitoring: it's time for routine quantitative assessment. Anesthesiology 2003 May;98(5):1037-1039. [Medline: 12717121] 
13. Kopman AF. Managing neuromuscular block: where are the guidelines? Anesth Analg 2010 Jul;111(1):9-10. [doi: 10.1213/ANE.0b013e3181cdb0a5] [Medline: 20576960]

14. Viby-Mogensen J, Claudius C. Evidence-based management of neuromuscular block. Anesth Analg 2010 Jul;111(1):1-2. [doi: 10.1213/ANE.0b013e3181e1d94c] [Medline: 20576957]

15. Avidan MS, Stevens TW. The diving bell and the butterfly. Br J Anaesth 2015 Jul;115 Suppl 1:i8-i10. [doi: 10.1093/bja/aev143] [Medline: 26174306]

16. Grayling M, Sweeney BP. Recovery from neuromuscular blockade: a survey of practice. Anaesthesia 2007 Aug;62(8):806-809 [FREE Full text] [doi: 10.1111/j.1365-2044.2007.05101.x] [Medline: 17635429]

17. Naguib M, Kopman AF, Lien CA, Hunter JM, Lopez A, Brull SJ. A survey of current management of neuromuscular block in the United States and Europe. Anesth Analg 2010 Jul;111(1):110-119. [doi: 10.1213/ANE.0b013e3181c07428] [Medline: $\underline{19910616]}$

18. Sorgenfrei IF, Viby-Mogensen J, Swiatek FA. [Does evidence lead to a change in clinical practice? Danish anaesthetists' and nurse anesthetists' clinical practice and knowledge of postoperative residual curarization]. Ugeskr Laeger 2005 Oct 10;167(41):3878-3882. [Medline: 16221428]

19. Thomsen JL, Nielsen CV, Palmqvist DF, Gätke MR. Premature awakening and underuse of neuromuscular monitoring in a registry of patients with butyrylcholinesterase deficiency. Br J Anaesth 2015 Jul;115 Suppl 1:i89-i94. [doi:

10.1093/bja/aev103] [Medline: 26174307]

20. Thomsen JL, Nielsen CV, Eskildsen KZ, Demant MN, Gätke MR. Awareness during emergence from anaesthesia: significance of neuromuscular monitoring in patients with butyrylcholinesterase deficiency. Br J Anaesth 2015 Jul;115 Suppl 1:i78-i88. [doi: 10.1093/bja/aev096] [Medline: 26174305]

21. Todd MM, Hindman BJ, King BJ. The implementation of quantitative electromyographic neuromuscular monitoring in an academic anesthesia department. Anesth Analg 2014 Aug;119(2):323-331. [doi: 10.1213/ANE.0000000000000261] [Medline: 24878683]

22. Baillard C, Clec'h C, Catineau J, Salhi F, Gehan G, Cupa M, et al. Postoperative residual neuromuscular block: a survey of management. Br J Anaesth 2005 Nov;95(5):622-626. [doi: 10.1093/bja/aei240] [Medline: 16183681]

23. Cook DA, Levinson AJ, Garside S, Dupras DM, Erwin PJ, Montori VM. Internet-based learning in the health professions: a meta-analysis. JAMA 2008 Sep 10;300(10):1181-1196. [doi: 10.1001/jama.300.10.1181] [Medline: 18780847]

24. McGaghie WC. Medical education research as translational science. Sci Transl Med 2010 Feb 17;2(19):19cm8. [doi: 10.1126/scitranslmed.3000679] [Medline: 20371485]

25. Wagner AK, Soumerai SB, Zhang F, Ross-Degnan D. Segmented regression analysis of interrupted time series studies in medication use research. J Clin Pharm Ther 2002 Aug;27(4):299-309. [Medline: 12174032]

26. Campbell MK, Piaggio G, Elbourne DR, Altman DG. Consort 2010 statement: extension to cluster randomised trials. BMJ 2012;345:e5661. [Medline: 22951546]

27. Yavner SD, Pusic MV, Kalet AL, Song HS, Hopkins MA, Nick MW, et al. Twelve tips for improving the effectiveness of web-based multimedia instruction for clinical learners. Med Teach 2015 Mar;37(3):239-244. [doi: 10.3109/0142159X.2014.933202] [Medline: 25109353]

28. Cook DA, Levinson AJ, Garside S, Dupras DM, Erwin PJ, Montori VM. Instructional design variations in internet-based learning for health professions education: a systematic review and meta-analysis. Acad Med 2010 May;85(5):909-922. [doi: 10.1097/ACM.0b013e3181d6c319] [Medline: 20520049]

29. Cook DA, Triola MM. What is the role of e-learning? Looking past the hype. Med Educ 2014 Sep;48(9):930-937. [doi: 10.1111/medu.12484] [Medline: 25113119]

\section{Abbreviations}

TOF: train-of-four

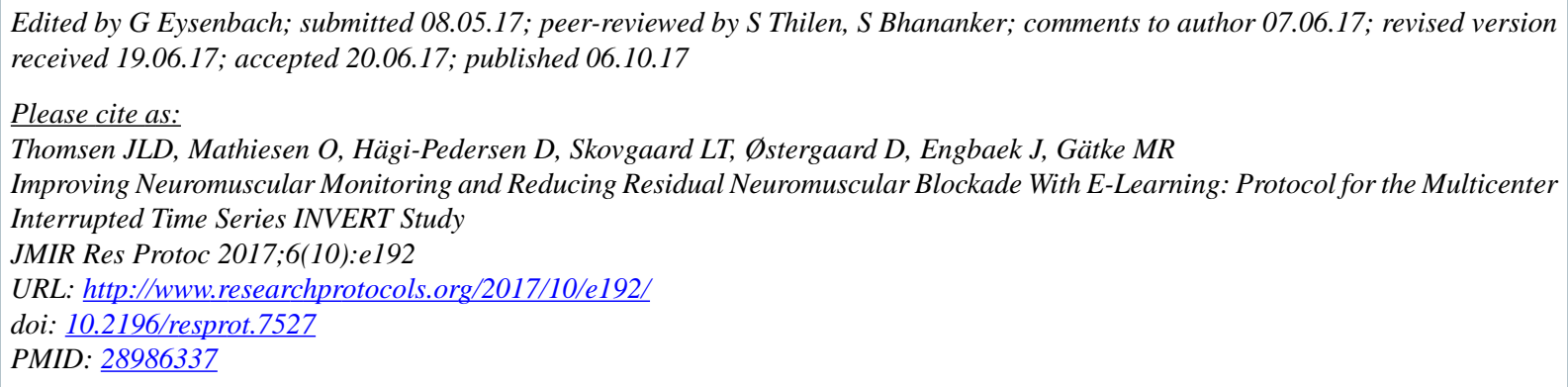


C Jakob Louis Demant Thomsen, Ole Mathiesen, Daniel Hägi-Pedersen, Lene Theil Skovgaard, Doris Østergaard, Jens Engbaek, Mona Ring Gätke. Originally published in JMIR Research Protocols (http://www.researchprotocols.org), 06.10.2017. This is an open-access article distributed under the terms of the Creative Commons Attribution License (https://creativecommons.org/licenses/by/4.0/), which permits unrestricted use, distribution, and reproduction in any medium, provided the original work, first published in JMIR Research Protocols, is properly cited. The complete bibliographic information, a link to the original publication on http://www.researchprotocols.org, as well as this copyright and license information must be included. 